\title{
Contabilidad de gestión: efecto en los factores clave del éxito competitivo en el sector cooperativo
}

\author{
Adalberto Escobar Castillo \\ Gabriel Velandia Pacheco \\ Evaristo Navarro Manotas
}

RESUMEN: Las cooperativas cobran cada vez más relevancia por su participación en el mercado y para ser competitivas, sus directivos deben sortear las exigencias de entornos dinámicos, tomando decisiones a partir de información extensa, ambigua y cambiante. Por otro lado, se entiende a la contabilidad de gestión como la herramienta que apoya a la gerencia en este proceso, sin embargo, en la literatura no existe claridad sobre su contribución a la competitividad. En consecuencia, el objetivo es explicar la incidencia de las dimensiones de la contabilidad de gestión en los factores clave del éxito competitivo de las cooperativas multiactivas. Para ello, se desarrolla una investigación de enfoque cuantitativo, alcance explicativo y diseño no experimental-transeccional de campo. Se aplicó un cuestionario con escala ordinal tipo Likert de seis opciones a 126 gerentes. Los resultados demuestran una influencia positiva de la amplitud, oportunidad e integración y negativa de la agregación de la información contable. Se concluye que, de manera general la contabilidad de gestión contribuye a la competitividad de las cooperativas multiactivas; sin embargo, resulta relevante la relación inversa de la agregación, la cual puede ser explicada tentativamente por la complejidad de este proceso, exigencia de tecnología especializada para tal fin y alta participación de micros y pequeñas empresas en la muestra, más sensibles a los altos costos de implementación. La implicación de estos resultados sugiere analizar la relación negativa de la agregación en los factores clave del éxito competitivo en el contexto de este estudio.

PALABRAS CLAVE: Contabilidad de gestión, competitividad, cooperativas multiactivas, factores clave del éxito competitivo, recursos y capacidades.

CLAVES ECONLIT: D23, D83, M10, M15.

Cómo citar este artículo / How to cite this article: ESCOBAR, A., VELANDIA, G. \& NAVARRO, E. (2020): "Contabilidad de gestión: efecto en los factores clave del éxito competitivo en el sector cooperativo", CIRIEC-España, Revista de Economía Pública, Social y Cooperativa, 99, 119-146. DOI: 107203/CIRIEC-E.99.14087.

Correspondencia: Adalberto Escobar Castillo, aescobar2@cuc.edu.co, ORCID ID: https://orcid.org/00000002-7116-8498 (autor de correspondencia); Gabriel Velandia Pacheco, gvelandi1@cuc.edu.co, ORCID ID: https://orcid.org/0000-0001-9009-3324, y Evaristo Navarro Manotas, enavarro3@cuc.edu.co, ORCID ID: https:// orcid.org/0000-0003-4549-502X. Universidad de la Costa CUC, Barranquilla-Colombia. 


\section{EXPANDED ABSTRACT}

\section{Management accounting: effect on key factors of competitive success in the cooperative sector}

\section{Contextualization and objective}

The importance of the cooperative sector for the economy has been demonstrated in the available literature (Comité Económico y Social Europeo, 2012; Mogrovejo et al., 2012; Confederación de Cooperativas de Colombia (CONFECOOP), 2017). However, these organizations also face turbulence in the market, where their managers are exposed to large volumes of information, which increases uncertainty in the decision-making process. This situation gives rise to the need to implement tools to efficiently manage the key factors of competitive success, with the aim of developing capabilities that can overcome the demands of the environment.

Management accounting is considered one of the tools used for this purpose, since its positive relationship with business competitiveness has been demonstrated (Simons, 1990; Ismail \& Isa, 2011). However, authors like Isa and Foong (2005) and Poporato (2015) point to a theoretical gap by indicating that, despite their contribution to reducing uncertainty in the decision-making process, their contribution to the development of competitive advantages through the management of key factors of competitive success is not clear, due to The market requires rapid responses and management accounting takes some time to process the information, thus affecting the ability to respond efficiently to critical situations of exogenous origin.

This situation leaves open the discussion on whether all aspects of management accounting contribute to business competitiveness. To resolve this theoretical discrepancy, it is considered necessary to dimension the management accounting in threads, to analyze its effect on the key factors of competitive success in the cooperative sector. Based on the above and given the importance of the cooperative sector in the economy, the objective of the study is to explain the incidence of the dimensions of management accounting on the key factors of competitive success in multi-activity cooperatives in Barranquilla (Colombia).

\section{Design and methodology}

An investigation of quantitative approach, explanatory scope and non-experimental design was carried out. Statistical tools such as measures of central tendency, dispersion, correlation coefficients (Pearson) and multiple linear regression are used to test the hypotheses. The population is made up of multi-activity cooperatives, which were chosen based on the definition of Colombian law and 
data provided by Cámara de Comercio of Barranquilla for the year 2017, resulting in a population of 186 multi-activity cooperatives. Applying a margin of error of $5 \%$, reliability coefficient of $95 \%$ and an estimated percentage of the sample of $50 \%$, a sample of 126 units was obtained, selected through simple random sampling. The technique used was the survey by means of a questionnaire applied to managers, which was validated by experts and whose Cronbach's alpha reliability index was 0.87 . The variables studied were measured using a Likert-type ordinal scale with six response options.

\section{Results, limitations, and implications}

As a relevant result, the negative and significant incidence of the aggregation of information stands out, with respect to the key factors of competitive success in the context of multi-active cooperatives, which can be tentatively explained by the high participation of SMEs in the population, complexity of the process and the sensitivity of these organizations to the high costs of applying aggregate information systems. Likewise, a positive and significant influence of the amplitude, opportunity, and integration on the key factors of competitive success is evident, suggesting that these variables add value to the information generated by management accounting to support the decision-making process.

However, these results refer to the Barranquilla's multi-active cooperatives and although they have allowed to delve into the particularities of this type of organizations, it has implications for a broader generalization of the results, since the solidarity sector is also made up of mutual and associative institutions, which have characteristics that differentiate them and that may be sensitive for the study. In this sense, this work presents limitations that constitute opportunities for future lines of research. For this reason, it is recommended to extend the study to these entities. On the other hand, it is suggested to develop investigations whose design is longitudinal with panel data, in order to have greater robustness in the data obtained.

\section{Conclusions and contributions}

It is possible to demonstrate the significant and positive influence of the dimensions of management accounting, breadth, opportunity, and integration on the key factors of competitive success. Thus highlighting the importance of the content and characteristics of accounting information that, within the framework of the theory of resources and capabilities, favors the development of competitive advantages through the identification of the key factors of competitive success, contributing to the reduction of the uncertainty in the decision-making process by managers. These findings allow us to recommend to the managers of the multi-active cooperatives that they focus their efforts on the manipulation of these variables, since they marked a significant and positive incidence on the key factors of competitive success.

Likewise, the results take on original value, because they provide empirical evidence that constitutes a significant contribution to the literature by proposing an explanation that presents the theoretical gap between the contributions of Isa and Foong (2005), Ismail and Isa (2011) and Poporato (2015); 
since it manages to demonstrate, on the one hand, the contribution of management accounting to the key factors of competitive success through breadth, opportunity and integration, and yet, on the other hand, manages to explain the reasons why it delays the ability to companies to provide timely responses to the demands of the environment, by revealing the significant and negative influence of aggregation within the process. This allows us to tentatively deduce that in the context of Barranquilla's multi-active cooperatives, the application of aggregated information systems is more expensive than giving up on them and this may be due to the aggregation is the dimension of management accounting it is more complicated when trying to detail the information by product lines, departments, periods and others, in multi-asset companies; making it more expensive and difficult to implement due to the requirement of greater resources to prepare it in a timely and reliable way to respond to the demands of the environment at the speeds it demarcates.

KEYWORDS: Management accounting, competitiveness, multi-active cooperatives, key factors of competitive success, resources and capabilities. 


\section{Introducción}

El cooperativismo está conformado por organizaciones sin ánimo de lucro pertenecientes al tercer sector, el cual surge como evolución del modelo de negocio tradicional capitalista, orientado a satisfacer necesidades de los agentes de mayor vulnerabilidad y aborda problemas de salud, vivienda, educación, trabajo y otros. En consecuencia, sus objetivos constituyen una intercepción entre las funciones del sector privado y público (Anheir \& Seibel, 1990; Bruque et al., 2002; Moyano et al., 2008; Lajara-Camilleri et al., 2017; Ladrón et al., 2018).

A nivel mundial, se evidencia que las cooperativas inciden significativamente en el bienestar socioeconómico. En la Unión Europea, generan 4.7 millones de empleos, a partir de la asociación de 102 millones de personas (Comité Económico y Social Europeo, 2012). En los Países Bajos, desarrollan el $83 \%$ de la producción agrícola y en España proveen el 21\% de asistencia sanitaria. Además, en Argentina 12 mil agrupan alrededor de 10 millones de asociados, en Brasil aportan cada año más de 100 mil empleos y en Colombia presentan un crecimiento estimado de 3.4 billones de pesos anuales (Mogrovejo et al., 2012; Confederación de Cooperativas de Colombia (CONFECOOP), 2017).

Ahora bien, aspectos como el desequilibrio macroeconómico, volatilidad del mercado, revolución microelectrónica, surgimiento de economías emergentes y desarrollo de Tecnologías de la Información y Comunicación (TIC), dieron lugar a entornos económicos turbulentos caracterizados por la alta incertidumbre en la toma de decisiones (Camillus \& Datta, 1991; Grant, 2003). Lo que establece un escenario que exige a las organizaciones gestionar factores clave para la creación de ventajas competitivas y habilidad para sortear exigencias del entorno, a través del desarrollo de capacidades y configuraciones de la cadena de valor (Penrose, 1959; Wernerfelt, 1984; Barney, 1991; Porter, 1991). En este contexto, para disminuir la incertidumbre en el proceso decisorio al enfrentarse a grandes volúmenes de información, los directivos deben contar con herramientas de apoyo. En esta línea, Simons (1990) y Caldera et al. (2007) consideran que los sistemas de contabilidad de gestión constituyen esta herramienta.

En coherencia, Ismail e Isa (2011) indican que la utilización de sistemas de contabilidad de gestión afecta positivamente la competitividad, sin embargo, Isa y Foong (2005) y Poporato (2015) identificaron una brecha o vacío teórico, al plantear que, a pesar de que reduce la incertidumbre en el proceso decisorio, no existe claridad acerca de su contribución al desarrollo de ventajas competitivas, a través de la gestión de los factores clave del éxito competitivo, debido a que, en entornos turbulentos caracterizados por el alto dinamismo, entorpece la capacidad de responder eficientemente a situaciones críticas de origen exógeno (Kattan et al., 2007; Mendoza, 2013). 
En este sentido, se identifica un gap de investigación al observar que, a pesar de que se ha demostrado la relación causal positiva entre la contabilidad de gestión y la competitividad, también se ha evidenciado que retrasa la capacidad de respuesta, lo cual, en entornos turbulentos, constituye una pérdida de ventaja competitiva. Esta situación deja abierta la discusión sobre si todos los aspectos de la contabilidad de gestión contribuyen a la competitividad empresarial. Para resolver esta discrepancia teórica, se considera necesario segmentar la contabilidad de gestión en subprocesos, para analizar su efecto sobre los factores clave del éxito competitivo. Con base en lo anterior y dada la importancia que presenta el sector cooperativo en la economía, se plantea como objetivo del estudio explicar la incidencia de las dimensiones de la contabilidad de gestión en los factores clave del éxito competitivo en las cooperativas multiactivas de Barranquilla (Colombia). Para lograr esto, se realizó una investigación de enfoque cuantitativo, alcance explicativo y diseño no experimental; utilizando como herramienta de análisis la regresión lineal múltiple, luego de aplicar encuestas en 126 unidades muestrales.

Como resultado relevante, se destaca la relación inversa de la agregación de la información, con respecto a los factores clave del éxito competitivo, lo que puede ser explicado tentativamente por la alta complejidad del proceso y la sensibilidad que poseen las PYME a los altos costos de la aplicación de sistemas de información agregado. Estos hallazgos proporcionan evidencia empírica que constituye un aporte significativo a la literatura. En lo que respecta a la estructura del trabajo, inicialmente se construyen las hipótesis a partir de los marcos teóricos existentes, luego se desarrollan los aspectos metodológicos para contrastarlas empíricamente. Además, se describen los resultados y se discuten a la luz de los cuerpos teóricos, con la finalidad de llegar a las conclusiones del estudio.

\section{Factores clave del éxito competitivo en el sector cooperativo}

Es consenso entre autores que, una ventaja competitiva logra ubicar a la empresa en una posición privilegiada con relación a su competencia. Con base en los aportes de Penrose (1959), Wernerfelt (1984) y Barney (1991), es definida como el resultado de configuraciones particulares y poco imitables de recursos y capacidades internas, útiles para sortear las exigencias del entorno. En coherencia, la competitividad es la vigencia e incremento de la participación en el mercado (Porter, 1991; Solleiro \& Castañón, 2012).

Para Paz et al. (2013) y Pal y Altay (2019), las diferencias entre el desempeño competitivo en un mismo sector económico, se explican desde los factores claves del éxito competitivo, los cuales son definidos por López (2007), como aquellos elementos indispensables para ser competitivo y que 
deben ser considerados al momento de formular las estrategias. Dichos componentes, se agrupan en tres dimensiones que representan variables macroeconómicas, sectoriales e internas (Acosta, 1999).

En lo que respecta al abordaje macroeconómico y sectorial, Porter (1991), López (2007), Porter (2008) y Llorente (2013), aseveran que los factores clave del éxito competitivo se relacionan con: regulaciones del mercado, tipologías de los factores de producción, característica de la demanda, presencia de industrias de apoyo (Clúster), poder de negociación de proveedores y clientes, barreras de entrada, bienes sustitutos, estructura de competencia, instituciones estatales, mercados financieros, tamaño de la economía y características geográficas.

Por su parte, los factores internos son aquellos sobre los cuales la organización posee mayor control y dependen principalmente de sus recursos y capacidades (Penrose, 1959; Wernerfelt, 1984; Barney, 1991). Desde esta perspectiva, Saavedra (2012) y Mora (2013) consideran que el análisis y medición de la competitividad, se ha enfocado en recolección de evidencia empírica relacionada con: innovación, productividad, gestión ambiental, posicionamiento estratégico, desempeño financiero y gestión del recurso humano. En la Tabla 1, se categoriza la revisión documental de los estudios que, basados en la teoría de los recursos y capacidades, relacionan los factores clave del éxito competitivo de carácter interno.

En síntesis, el estudio de la competitividad desde los factores clave del éxito competitivo interno, se aborda principalmente desde dos aspectos: operaciones del negocio y resultado de las operaciones. La primera, considera líneas relacionadas con los procesos administrativos, responsabilidad social, sistemas de información y recurso humano. La segunda, hace referencia a la innovación, productividad, posicionamiento estratégico y financiero. Lo cual es coherente y complementa lo expresado por Saavedra (2012) y Mora (2013). De esta manera, se evidencia una corriente de investigación, que vincula el estudio de la competitividad a partir de la teoría de recursos y capacidades.

Ahora bien, específicamente en el sector cooperativo, Bruque et al. (2002) expresan que las cooperativas son un tipo de organización societaria, en la cual, el socio además de ser proveedor de recursos y receptor de la cadena de valor funge como agente activo de los procesos de gestión empresarial. Por este motivo, los factores clave del éxito competitivo en este tipo de instituciones, provienen principalmente de elementos internos orientados a garantizar una adecuada relación con los socios. De hecho, Moyano et al. (2008) evidenciaron que, en la mayoría de los casos, los recursos intangibles provenientes de factores internos en las cooperativas determinan su éxito competitivo (Tabla 2). 


\section{Tabla 1. Estudio de la competitividad desde los factores} clave del éxito competitivo interno

\begin{tabular}{|c|c|c|c|}
\hline Categorías abiertas (Variables) & Categorías cerradas & Categorías emergentes & \multirow{26}{*}{ 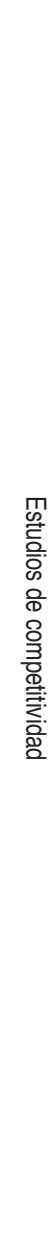 } \\
\hline Responsabilidad social empresarial & \multirow[t]{2}{*}{ Responsabilidad social } & \multirow{12}{*}{ Operación del negocio } & \\
\hline Gestión Medioambiental & & & \\
\hline Sistemas de información & \multirow[t]{2}{*}{ Sistemas de información } & & \\
\hline TIC & & & \\
\hline Gestión de recursos humanos & Recurso humano & & \\
\hline Planeación estratégica & \multirow{7}{*}{ Procesos administrativos } & & \\
\hline Aprovisionamiento & & & \\
\hline Comercialización & & & \\
\hline Capacidad de marketing & & & \\
\hline Capacidad directiva & & & \\
\hline Capacidad de innovación & & & \\
\hline Estrategias de comercialización & & & \\
\hline Gestión del conocimiento & \multirow{3}{*}{ Innovación } & \multirow{13}{*}{ Resultado de las operaciones } & \\
\hline Innovación & & & \\
\hline Adquisición de conocimiento & & & \\
\hline Costo & \multirow{4}{*}{ Productividad } & & \\
\hline Calidad & & & \\
\hline Tecnología & & & \\
\hline Eficiencia & & & \\
\hline Participación en el mercado & \multirow[t]{2}{*}{ Posicionamiento estratégico } & & \\
\hline Incursión en nuevos mercados & & & \\
\hline Contabilidad y finanzas & \multirow{4}{*}{ Financiero } & & \\
\hline Control financiero & & & \\
\hline Desempeño financiero & & & \\
\hline Rentabilidad & & & \\
\hline
\end{tabular}

FUENTE: Elaboración propia a partir de Bernal et al. (2012); Enríquez et al. (2013); Vázquez et al. (2014); Mora (2013); García y López (2014); Martínez et al. (2010); López-Gamero y Molina-Azorín (2016); Aguiar et al. (2016); Bloodgood (2019). 


\section{Tabla 2. Factores clave del éxito competitivo en las cooperativas}

\begin{tabular}{|c|c|}
\hline Autor & Factor clave del éxito competitivo \\
\hline Bruque et al. (2002) & Grado de lealtad de los asociados y Estructura de propiedad \\
\hline Montegut (2006) & $\begin{array}{l}\text { Adecuada dimensión; eficiencia en la utilización de los recursos tecnológicos; correcta } \\
\text { financiación; adaptación del servicio basado en la calidad; formación del recurso } \\
\text { humano }\end{array}$ \\
\hline Moyano et al. (2008) & $\begin{array}{l}\text { Capacidad de reacción ante los requerimientos del asociado; participación de los } \\
\text { asociados en los resultados; }\end{array}$ \\
\hline Nilsson et al. (2009) & Dimensión; Aspectos financieros e integración; \\
\hline Lajara-Camilleri et al. (2017) & Orientación al mercado e innovación \\
\hline Parilina y Sedakov (2020) & Coalición \\
\hline
\end{tabular}

FUENTE: Elaboración propia a partir de los autores citados.

Estos trabajos evidencian que en las cooperativas la competitividad puede ser alcanzada a través de la gestión de estos elementos; por lo tanto, en el contexto del estudio se utilizan para medir la variable factores clave del éxito competitivo. Para ello se define en primera instancia, el grado de lealtad de los asociados, como la disposición que presentan los socios en la aceptación de responsabilidades teniendo como prevalencia el bienestar social (Bruque et al. 2002; Villafañez, 2015). Por su parte, la estructura de propiedad hace referencia a la forma de organizarse para ejercer control sobre los gestores según su naturaleza y grado de concentración de los recursos, teniendo implicaciones directas sobre: forma de gestionar, decisiones de inversión, financiación y resultados (Del Orden \& Garmendina, 2008).

En cuanto a la adecuada dimensión, esta se refiere al tamaño de la cooperativa ajustado a su realidad socioeconómica, la cual le permite incrementar la cuota de participación en el mercado, impactando directamente en la competitividad (Montegut, 2006; Nilsson et al. 2009; Arcas et al., 2013). Por su parte, la orientación al mercado e innovación hacen alusión a la iniciativa de la organización de reconocer y aprovechar las nuevas tendencias del mercado, haciendo uso de la capacidad adaptación (Lajara-Camilleri et al., 2017).

En lo que respecta a los aspectos financieros, Montegut (2006) y Nilsson et al. (2009) consideran que el apalancamiento se convierte en un factor clave del éxito competitivo, cuando la cooperativa es capaz de generar fuentes de autofinanciación relacionadas con el capital social, reservas, fondos de destinación específica o aportes de la sección de crédito. Por otro lado, la eficiencia en la utilización de los recursos tecnológicos alude a la posición competitiva de la cooperativa a partir de la actualización y utilización eficiente de las herramientas tecnológicas. 
Asimismo, la adaptación del servicio basado en la calidad se refiere a la mejora continua de los procesos que contribuyen al incremento sostenido de ventajas competitivas. En la misma línea, la capacidad de reacción ante los requerimientos de los asociados hace referencia a la priorización del capital relacional, considerando la participación activa de los asociados en la toma de decisiones. Por su parte, la formación del recurso humano consiste en la gestión planificada de las capacidades y habilidades de los integrantes de una organización (Montegut, 2006; Díaz \& Márquez, 2005; Moyano et al., 2008).

Finalmente, la participación de los asociados en los resultados hace referencia al nivel de involucramiento de los socios con los resultados organizacionales, sustentado bajo la premisa de que se mejoran los índices de competitividad cuando estos poseen mayor participación (Moyano et al., 2008). Además, la integración y coalición son las actividades usadas para precisar objetivos organizacionales, considerando la convergencia entre los distintos departamentos u otras instituciones solidarias (Chenhall \& Morris, 1986; Parilina y Sedakov, 2020).

\section{Incidencia de la contabilidad de gestión en los factores clave del éxito competitivo en cooperati- vas}

En coherencia con Velandia (2010) y Velandia et al. (2018), la contabilidad puede entenderse como una tecnología, la cual utiliza un conjunto de técnicas con el fin de generar representaciones sintéticas de los procesos de intercambios de recursos, mediante procedimientos formalizados que buscan racionalidad en sus representaciones. No obstante, la utilidad de la información no posee carácter universal, sino que se encuentra supeditada a las necesidades del usuario. Estos pueden ser: Estado, clientes, proveedores, inversores, comunidad en general, fisco, gerente, entre otros.

De lo anterior se deduce que, la elaboración y presentación de la información contable, se encuentra influenciada por el usuario, por tanto, al existir distintas necesidades, pueden definirse múltiples formas de representar los hechos económicos, y a su vez, diversos criterios para calificarla como útil para la toma decisiones. Esta diversidad se minimiza con los procesos de estandarización normativa de la información de carácter público, lo cual si bien, genera grandes ventajas a la hora de comunicar socialmente la situación financiera de una organización, permite que se le reste importancia a la construcción de información útil para la gerencia y otro tipo de usuarios más específicos, que muchas veces debe desprenderse de lo normativo para lograr la relevancia requerida.

Partiendo de estos supuestos, la contabilidad de gestión propende por la construcción y presentación de información relevante para la administración (Chenhall \& Morris, 1986). Busca satisfacer las 
necesidades particulares de los directivos, suministrando información oportuna y suficiente con orientación interior, a corto plazo y cuantificada, para evaluar la eficiencia de las operaciones internas y externas (Johnson \& Kaplan, 1987). Además, contribuye a la coordinación entre agentes y principales en los procesos de delegación y control (Porporato, 2015).

Asimismo, Chenhall \& Morris (1986); Bouwens \& Abernethy (2000) proponen cuatro dimensiones para la contabilidad de gestión: amplitud, oportunidad, agregación e integración. La amplitud hace referencia al horizonte temporal, orientación y cuantificación de la información. La oportunidad a la disponibilidad, pertinencia y confiabilidad de la información para la toma de decisiones. Por su parte la agregación denota suministro de información agrupada en líneas básicas de producto, departamento o periodos de tiempo. Finalmente, la integración hace alusión a la disponibilidad de información que interrelacione distintos departamentos y/o servicios, para contribuir a la dirección simultánea y coordinada, de todas las secciones de la empresa.

En este orden de ideas, Naranjo (2005), con base en el trabajo realizado por Chenhall \& Morris (1986), organiza la aplicación del sistema de contabilidad de gestión según sus formas tradicionales e innovadoras. Este trabajo se considera pertinente para extraer de él los principales aspectos de cada una de las dimensiones de la contabilidad de gestión presentada por Chenhall \& Morris (1986) y Bouwens \& Abernethy (2000), tal como se muestra en el Tabla 3.

\section{Tabla 3. Características de la contabilidad de gestión}

\begin{tabular}{|l|l|l|}
\hline $\begin{array}{l}\text { Dimensión de la contabili- } \\
\text { dad de gestión }\end{array}$ & Tradicional & Innovador \\
\hline Amplitud & $\begin{array}{l}\text { Centrada en hechos internos } \\
\text { Cuantificada en términos monetarios } \\
\text { Relacionada con datos históricos }\end{array}$ & $\begin{array}{l}\text { Centrada en el entorno externo } \\
\text { Cuantificada en términos no monetarios y } \\
\text { monetarios } \\
\text { Relacionada con datos futuros (probabilidad) }\end{array}$ \\
\hline Oportunidad & $\begin{array}{l}\text { Bajo grado de reportes } \\
\text { Orientación cortoplacista }\end{array}$ & $\begin{array}{l}\text { Información dinámica y exógena } \\
\text { Orientación a largo plazo }\end{array}$ \\
\hline Agregación & $\begin{array}{l}\text { Información poco agregada } \\
\text { Datos poco procesados }\end{array}$ & $\begin{array}{l}\text { Información muy agregada por periodo de ti- } \\
\text { empo y áreas funcionales }\end{array}$ \\
\hline Integración & $\begin{array}{l}\text { No coordinada con las distintas secciones } \\
\text { de la organización } \\
\text { Sin objetivos precisos para las actividades } \\
\text { e interrelacionada con los departamentos }\end{array}$ & $\begin{array}{l}\text { Objetivos precisos para las actividades y su } \\
\text { interrelación con los departamentos } \\
\text { Informes sobre las interacciones dentro de } \\
\text { cada subunidad }\end{array}$ \\
\hline \multicolumn{2}{|l|}{} \\
\hline
\end{tabular}

FUENTE: Elaboración propia a partir de Chenhall \& Morris (1986); Bouwens \& Abernethy (2000), y Naranjo (2005). 
Aunque no existe claridad en la relación entre contabilidad de gestión y factores clave del éxito competitivo (Isa \& Foong, 2005; Porporato, 2015), trabajos como los de Ghasemi et al. (2015); Ismail et al. (2018) y Terdpaopong et al. (2019), demostraron que estas variables se vinculan positivamente, constituyéndose en una aproximación a su comprensión. A partir de estos resultados, se puede inferir que, la contabilidad de gestión genera información que afecta positivamente los procesos directivos encaminados a gestionar los factores clave del éxito competitivo (Figura 1). En consecuencia, este trabajo contribuye a la literatura al explicar los efectos de las dimensiones de la contabilidad de gestión destacadas por Chenhall \& Morris (1986); Bouwens \& Abernethy (2000) y Naranjo (2005), sobre los factores clave del éxito competitivo en las cooperativas.

Ahora bien, en cuanto a la amplitud de la información, Thomsen (1995) y Cruz-Cázares et al. (2018) evidencian que esta contribuye al monitoreo y seguimiento en los procesos directivos, impactando significativamente la efectividad, tanto de la operación del negocio, como de sus resultados. En la misma línea, Weiss et al. (2008) indican que la amplitud afecta los juicios objetivos de la información. Asimismo, Twedt (2016) y Crespo et al. (2019) demostraron que aspectos de la amplitud como cultura, estrategia y estructura organizacional, poseen un efecto moderador en la toma de decisiones. Con base en estos antecedentes, los cuales destacan los efectos de la amplitud sobre aspectos relacionados con los factores clave del éxito competitivo de la Tabla 1, se puede expresar que:

$\mathbf{H}_{1}$ : La amplitud de la información generada por la contabilidad de gestión, afecta positivamente los factores clave del éxito competitivo de las cooperativas.

Por su parte, Moll \& Yigitbasioglu (2019) demuestran el impacto en los sistemas contables surgido de la implementación de tecnologías, a través de la mejora significativa de la visibilidad financiera y la intervención oportuna. Así mismo, Siqani \& Vokshi (2019) proporcionan evidencia del impacto significativo de la información contable sobre la eficacia de empresas públicas; afectando la calidad de la información financiera, toma de decisiones, sistema de control, desempeño, estabilidad financiera y transacciones, lo cual se vería retrasado si el sistema de información no fuera oportuno. Por otra parte, Rubio \& Unsal (2020) señalan que la oportunidad de la información hace más efectiva la estabilidad financiera. Asimismo, Hong et al. (2019) comprobaron que la información oportuna está asociada con los rendimientos contemporáneos del mercado y las revisiones de pronósticos de analistas. Además, Liao et al. (2019) estudian los efectos de la oportunidad de la información en la calidad de las decisiones del sector agricultor en países en desarrollo, demostrando su relevancia. Todos estos antecedentes vinculan la oportunidad de la información con algún aspecto definido como factor clave del éxito competitivo de la Tabla 1. En este orden de ideas, se puede expresar que:

$\mathbf{H}_{2}$ : La oportunidad de la información generada por la contabilidad de gestión, afecta positivamente los factores clave del éxito competitivo de las cooperativas. 
Siguiendo la misma corriente, Kajüter \& Nienhaus (2017), Sharovatova et al. (2018) y Govdya \& Khromova (2018) y Bens et al. (2018) presentan evidencia que sustenta la influencia significativa de las políticas de agregación de la contabilidad de gestión, en la disminución de la asimetría de información, aumentando su transparencia y confianza, lo que mejora la calidad de los informes contables disponibles para la dirección de las empresas innovadoras en entornos globalizados, así como, la evaluación de los objetos de gestión, facilitando la toma de decisiones. Además, Mangaliso (1995) demostró que la agregación de la información tiene una relación significativa con la disminución de la incertidumbre. Por su parte, Chen et al. (2020) comprobaron que las organizaciones con información contable agregada presentan menos restricciones financieras, de lo que se esperaría una posición competitiva en el mercado; de esta manera, se destaca la relación existente entre la agregación de la información y los factores clave del éxito competitivo. En este sentido, se puede aseverar que:

$\mathbf{H}_{3}$ : La agregación de la información generada por la contabilidad de gestión, afecta positivamente los factores clave del éxito competitivo de las cooperativas.

Asimismo, los reportes integrados se basan en los puntos fuertes de la contabilidad, tales como materialidad, confiabilidad, comparabilidad, relevancia, asegurabilidad y una evidencia cuantitativa robusta, para dar lógica al discurso de la sostenibilidad y orientar la toma de decisiones. Contribuyendo a una dirección simultánea y coordinada, al generar mejor visibilidad e identificación de lo financiero; ofreciendo adicionalmente un enfoque multifacético del desempeño organizacional, a través de la convergencia de información de distintos departamentos, servicios u organizaciones (Theeuwes \& Adriaansen, 1994; Hassan et al., 2019). Alineadamente, los trabajos de Angeles (2010); Manyaeva et al. (2016); Prodanova (2019) y Fomin y Potokina (2020), relacionados con la integración de los sistemas de contabilidad de gestión y la consolidación de una plataforma informática única, evidencian la tendencia a integrar la información, para ampliar su uso en la toma de decisiones en empresas que operan en entornos turbulentos, constituyendo la base para desarrollar la estrategia empresarial; lo que contribuye a la mejora de la productividad. Lo anterior, evidencia la relación existente entre la integración de la información contable y los factores clave del éxito competitivo. Bajo estos preceptos se puede expresar que:

$\mathbf{H}_{4}$ : La integración de la información generada por la contabilidad de gestión, afecta positivamente los factores clave del éxito competitivo de las cooperativas. 


\section{Figura 1. Modelo conceptual}

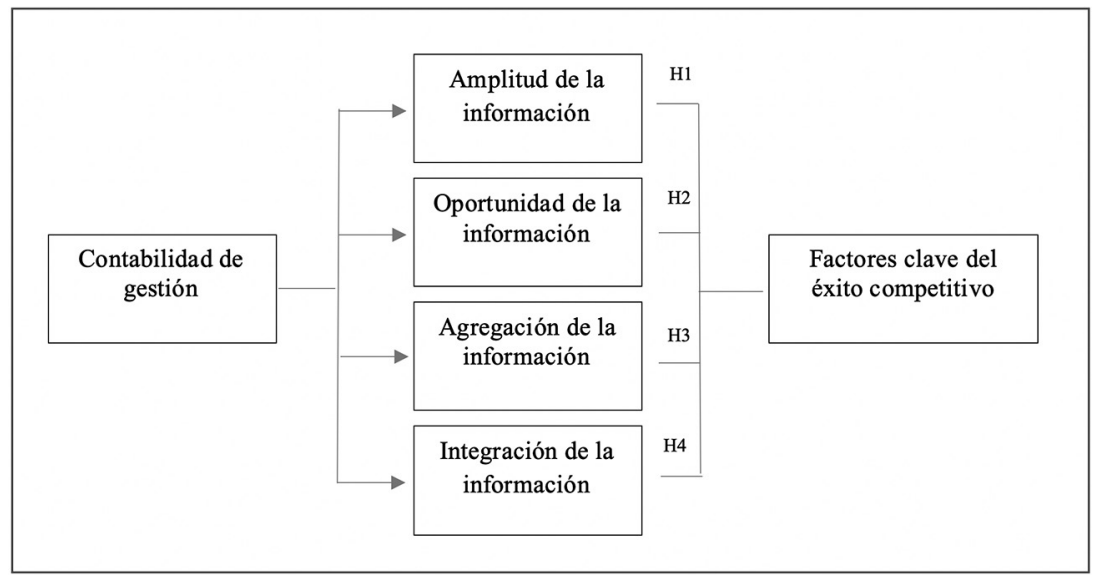

FUENTE: Elaboración propia a partir de los fundamentos teóricos.

\section{Aspectos metodológicos}

En coherencia con Hernández et al. (2014) la investigación se enmarcó en el paradigma positivista, debido a que se construyó el objeto de estudio con anterioridad a la verificación empírica, tomando como referencia las bases teóricas relacionadas con la contabilidad de gestión y factores clave del éxito competitivo en el contexto de las cooperativas; por otro lado, para la prueba de hipótesis se utilizaron herramientas estadísticas como medidas de tendencia central, dispersión, coeficientes de correlación (Pearson) y regresión lineal múltiple, apoyados en el programa Statistical Package for the Social Sciences (SPSS) versión 24. Posee un alcance explicativo y diseño no experimental de corte transversal de campo.

La población está constituida por las cooperativas multiactivas, las cuales son definidas en Colombia por la Ley 79 (Congreso de la República de Colombia, 1988), como aquellas cuyo objeto es satisfacer múltiples necesidades, ubicándose en diferentes sectores económicos, bajo la responsabilidad de una sola persona jurídica de naturaleza solidaria. El estudio se enfoca en estas instituciones, debido a la relevancia social evidenciada por el amplio rango de beneficios que sus actividades generan (Moriano, 2008). De hecho, Silva (2008) expresa que su importancia radica, en que representan cerca del $20 \%$ del total del sector solidario y han presentado una tendencia de constante crecimiento. 
De acuerdo a la base de datos suministrada por la Cámara de Comercio de la ciudad de Barranquilla en el año 2017, la población que cumple los criterios de inclusión fue de 186 cooperativas multiactivas. Utilizando como parámetros muestrales, un margen de error del $5 \%$, coeficiente de confiabilidad del $95 \%$ y un porcentaje estimado de la muestra del $50 \%$, resulta una muestra de 126 unidades, las cuales fueron seleccionadas a través del muestreo probabilístico aleatorio simple. Como técnica, se empleó la encuesta por medio de la aplicación de un cuestionario a los directivos. Para la medición de las variables, se definió una escala ordinal tipo Likert de seis opciones, la cual es ampliamente usada en estudios previos, referentes a la contabilidad de gestión y factores clave del éxito competitivo (Tabla 4). Desde el punto de vista metodológico, se justifica esta escala, debido a que, en el contexto de este trabajo, es utilizada para medir en los gerentes el nivel de concordancia con las afirmaciones presentadas, ya que trabajan con información revelada por la contabilidad de gestión para tomar decisiones, con respecto a los factores clave del éxito competitivo; resultando coherente con los indicadores empíricos definidos en la operacionalización de las variables.

\section{Tabla 4. Escalas utilizadas en la medición de las variables}

\begin{tabular}{|c|c|c|c|}
\hline Variable & Escala & Valores & Antecedentes \\
\hline $\begin{array}{l}\text { Factores clave del éxito } \\
\text { competitivo }\end{array}$ & \multirow{2}{*}{ Ordinal } & \multirow{2}{*}{$\begin{array}{c}\text { 6: Total acuerdo } \\
\text { 5: Acuerdo } \\
\text { 4: Moderado acuerdo } \\
\text { 3: Moderado desacuerdo } \\
\text { 2: Desacuerdo } \\
\text { 1: Total desacuerdo }\end{array}$} & $\begin{array}{l}\text { Martínez et al. (2010); López-Gamero et al. (2010); } \\
\text { López-Gamero \& Molina-Azorín (2016). }\end{array}$ \\
\hline Contabilidad de gestión & & & $\begin{array}{c}\text { Simons (1995); Naranjo-Gil \& Hartmann (2007); } \\
\text { López-Valeiras et al. (2016) }\end{array}$ \\
\hline
\end{tabular}

FUENTE: Elaboración propia.

Para medir la confiabilidad del instrumento, se utilizó el índice alfa de Cronbach, el cual es una medida ponderada de las correlaciones existentes entre las variables, calculado a partir de las varianzas; sus resultados, se sitúan entre cero (0) y uno (1), presentando mayor confiabilidad cuando su valor se aproxime al rango más alto. Una vez aplicada la prueba piloto al 10\% de la población, el índice de confiabilidad alfa de Cronbach se ubicó en 0.87 , lo que garantiza coherencia y consistencia en los resultados (Hernández et al., 2014).

Por otro lado, el instrumento se estructuró a partir de la operacionalización de las variables, apoyado en el marco teórico definido, obteniendo un total de 64 afirmaciones que fueron validadas inicialmente a través del juicio de expertos. El cuestionario, fue entregado a los 126 gerentes de las cooperativas, a través de dos vías: personal y correo electrónico, en donde se pidió señalaran, entre las opciones de respuesta, el nivel de concordancia con las afirmaciones presentadas. La tasa de respuesta fue del $100 \%$. 
En Colombia la Ley 905 (Congreso de la República de Colombia, 2004), establece los parámetros para definir el tamaño de las organizaciones a partir de dos criterios: volumen de activos y número de trabajadores. Con base en estas disposiciones, la muestra está constituida en $24 \%$ por micro, $40 \%$ pequeñas, $24 \%$ medianas y $12 \%$ grandes. Sin embargo, esta variable no fue controlada en el análisis, debido a que Montegut (2006) y Nilsson et al. (2009) demostraron que la dimensión (tamaño) constituye un factor clave del éxito competitivo, por lo que esta fue tenida en cuenta dentro de los indicadores que integran la variable dependiente.

Para verificar las hipótesis planteadas, se empleó un modelo de regresión lineal múltiple, que en términos de Gajarati y Porter (2009), constituye una estimación indicativa de la dependencia entre variables. Las hipótesis se contrastaron a partir del p-valor. Se busca un patrón adecuado, a partir de los factores independientes relacionados con la amplitud, oportunidad, agregación e integración de la información generada por la contabilidad de gestión. En términos matemáticos, se puede representar por:

$$
F C E_{t}=\beta_{0}+\beta_{1} A M P_{t}+\beta_{2} O P O_{t}+\beta_{3} A G R_{t}+\beta_{4} I N T_{t}+\varepsilon
$$

Dónde:

FCE: Factores clave del éxito competitivo

AMP: Amplitud de la información

OPO: Oportunidad de la información

AGR: Agregación de la información

INT: Integración de la información

Finalmente, a través de la prueba de normalidad Kolmogorov-Smirnov se evidenció que la distribución de los errores es normal. Por otra parte, se demuestra el cumplimiento del supuesto de linealidad, así como la inexistencia de auto correlación y colinealidad en las variables explicativas. Además, el modelo cumple con los criterios de homocedasticidad. Se observa que el modelo explica en un $74,9 \%$ la variable dependiente, que, una vez corregido por el efecto de la muestra y de las variables independientes, resulta ser $69,9 \%$. Asimismo, el error típico de la estimación de 0.4675 y el valor del estadístico Durbin Watson de 1.550, indican que se cumplen los parámetros de independencia establecidos. 


\section{Resultados y discusión}

En lo que respecta a la variable FCE, las medidas de tendencia central y dispersión (media de 4.08 y desviación típica de 1.115) demuestran que los factores clave del éxito competitivo son tenidos en cuenta para definir las estrategias competitivas (Tabla 5). Ahora bien, la adaptación del servicio basado en la calidad, grado de lealtad de los asociados y estructura de propiedad, trabajados por Bruque et al (2002), Montegut (2006) y Moyano et al (2008), presentan promedios aritméticos de 2.24 y 2.29 respectivamente, lo que sugiere que, en este tipo de organizaciones no son considerados importantes, a pesar de que estos autores han demostrado sus efectos sobre la competitividad.

Además, las variables explicativas AMP, OPO, AGR e INT presentan medias aritméticas ubicadas en $4.44 ; 4.76 ; 4.72$ y 4.68 respectivamente, lo que indica tendencias de respuesta superiores a 4 (Moderado Acuerdo). En este sentido, la evidencia estadística demuestra que, los gerentes le asignan importancia a la contabilidad de gestión. Esto puede explicarse desde los planteamientos de Chenhall \& Morris (1986), Johnson \& Kaplan (1987) y Porporato (2015), quienes consideran que esta se constituye en un apoyo a los procesos administrativos al revelar información que disminuye el riesgo en la toma de decisiones.

\section{Tabla 5. Estadística descriptiva}

\begin{tabular}{|c|c|c|c|}
\hline & Media & Desviación típica & $\mathrm{N}$ \\
\hline FCE & 4.08 & 1.115 & 126 \\
\hline AMP & 4.44 & 1.083 & 126 \\
\hline OPO & 4.76 & 1.052 & 126 \\
\hline AGR & 4.72 & 1.100 & 126 \\
\hline INT & 4.68 & 1.069 & 126 \\
\hline
\end{tabular}

FUENTE: Elaboración propia a partir de los datos arrojados por la encuesta aplicada (2017).

Se evidencia, una correlación positiva y significativa al $1 \%$ entre FCE y las variables AMP y OPO. Por otro lado, se observa la misma relación entre FCE e INT con una significancia del $5 \%$. Además, la relación entre FCE y AGR no es significativa (Tabla 6). Estos resultados sugieren la poca incidencia que poseen las políticas de agregación de la información, en la gestión de los factores clave del éxito competitivo en las cooperativas; contrario a los planteamientos de Naranjo (2005), Kajüter \& Nienhaus (2017), Sharovatova et al. (2018) y Govdya \& Khromova (2018). 
Tabla 6. Correlaciones

\begin{tabular}{|c|c|c|}
\hline \multicolumn{2}{|c|}{ AMP } & Factores Clave del éxito competitivo \\
\cline { 2 - 3 } & Correlación de Pearson & $0.625^{*}$ \\
\hline \multirow{2}{*}{ OPO } & Sig. & 0.000 \\
\cline { 2 - 3 } & Correlación de Pearson & $0.656^{*}$ \\
\hline \multirow{2}{*}{ AGR } & Sig. & 0,000 \\
\cline { 2 - 3 } & Correlación de Pearson & 0.291 \\
\hline \multirow{2}{*}{ INT } & Sig. & 0.079 \\
\cline { 2 - 3 } & Correlación de Pearson & $0.442^{*}$ \\
\hline \multirow{2}{*}{ Sig. Nota: ${ }^{*} p<0.05^{* *} p<0.01}$. & 0.014 \\
\hline
\end{tabular}

FUENTE: Elaboración propia a partir de los datos arrojados por la encuesta aplicada (2017).

Se observa en la Tabla 7 la incidencia positiva y significativa al $5 \%$ de la amplitud de la información, en los factores clave del éxito competitivo de las cooperativas ( $\beta$ : 0.207, p-valor: 0.033). Coherente con los planteamientos de Thomsen (1995), Weiss et al. (2008), Twedt (2016), Cruz-Cázares et al. (2018), Crespo et al. (2019), estos resultados sugieren la contribución de la amplitud, agregando valor en la utilidad de la información generada por la contabilidad de gestión en la toma de decisiones, afectando positivamente los factores clave del éxito competitivo de las cooperativas. En este sentido, se acepta la $\mathrm{H}_{1}$.

De igual forma, se advierte una influencia positiva y significativa al $1 \%$ de la oportunidad de la información, sobre los factores clave del éxito competitivo de las cooperativas ( $\beta: 0.330, p$-valor: 0.006$)$. A partir de estos resultados, coherentes con Moll \& Yigitbasioglu (2019), Siqani \& Vokshi (2019), Hong et al. (2019), Liao et al. (2019) y Rubio \& Unsal (2020), se infiere que la información oportuna posee mayor valor por parte de la gerencia en la toma de decisiones, lo cual influye en el desempeño organizacional, afectando positivamente los factores clave del éxito competitivo de las cooperativas. En consecuencia, se confirma la $\mathrm{H}_{2}$

Asimismo, la relación causal entre la agregación de la información y los factores clave del éxito competitivo de las cooperativas es negativa y significativa al $5 \%$ ( $\beta:-0.217, p$-valor: 0.039 ). Esto puede ser explicado tentativamente por la importante participación de PYME en el sector solidario en Colombia, el cual según CONFECOOP (2017) corresponde al 98.75\%, lo que es coherente con la muestra objeto de estudio. Además, por la multiactividad de las empresas estudiadas, lo que hace que sea más compleja e ineficiente la implementación de información agregada. 
Por su parte, Velandia et al. (2016) plantean que, las PYME generalmente se caracterizan por llevar sistemas de gestión sencillos o incluso informales, sin embargo, suficientes para su realidad operacional, los cuales, sumados a la escasez de recursos, incide en que se encuentren más expuestas a las exigencias que genera el diseño e implementación de sistemas de información agregados, ya que, dicha actividad requiere grandes esfuerzos de inversión en tecnologías y capacitación (Prieto at al., 2007). Además, la capacidad de las cooperativas multiactivas de ejercer múltiples actividades eleva el nivel de complejidad de los sistemas de información requeridos. Con base en estos resultados se rechaza la $\mathrm{H}_{3}$ refutando los hallazgos de los trabajos de Mangaliso (1995), Kajüter \& Nienhaus (2017), Bens et al. (2018), Sharovatova et al. (2018), Govdya \& Khromova (2018) y Chen et al. (2020) en el contexto de las cooperativas multiactivas.

Finalmente, los resultados constituyen evidencia empírica que soporta la $\mathrm{H}_{4}$, debido a que se observa una incidencia positiva al 1\% de significancia de la integración de la información, sobre la gestión de los factores clave del éxito competitivo en las cooperativas. Corroborando los hallazgos de Theeuwes \& Adriaansen, (1994), Angeles (2010), Manyaeva et al. (2016), Hassan et al. (2019), Prodanova et al. (2019) y Fomin y Potokina (2020). Estos resultados, permiten aseverar que la integración agrega valor a la información, afectando positivamente los factores clave del éxito competitivo de las cooperativas, por lo que se acepta la $\mathrm{H}_{4}$

\section{Tabla 7. Modelo de regresión}

\begin{tabular}{|c|c|c|c|c|c|}
\hline Hipótesis & Modelo & Std. beta & Std. error & Sig. & Decisión \\
\hline & (Constante) & 0.613 & 0.697 & 0.389 & \\
\hline $\mathrm{H}_{1}$ & AMP & 0.207 & 0.090 & $0.033^{*}$ & Aceptada \\
\hline $\mathrm{H}_{2}$ & OPO & 0.330 & 0.106 & $0.006^{*}$ & Aceptada \\
\hline $\mathrm{H}_{3}$ & AGR & -0.217 & 0.098 & $0.039^{*}$ & Rechazada \\
\hline $\mathrm{H}_{4}$ & INT & 0.548 & 0.102 & $0.000^{*}$ & Aceptada \\
\hline \multicolumn{7}{|c|}{ Nota: ${ }^{*} \mathrm{p}<0.05^{* *} \mathrm{p}<0.01}$. \\
\hline
\end{tabular}

FUENTE: Elaboración propia a partir de los datos arrojados por la encuesta aplicada (2017).

Los hallazgos contribuyen a la literatura existente, en torno a la discusión sobre los aportes que realiza la contabilidad de gestión a los factores clave del éxito competitivo de las cooperativas multiactivas, profundizando en las dimensiones amplitud, oportunidad, agregación e integración propuestas por Chenhall \& Morris (1986); Bouwens \& Abernethy (2000) y Naranjo (2005) como factores que la diferencian de la contabilidad financiera. A pesar de que la agregación de información posee un efecto negativo en este tipo de empresas, los resultados de esta investigación son coherentes parcialmente con dichos planteamientos y el modelo permite vincular a la contabilidad de gestión, como un factor interno que agrega valor a la información utilizada en los procesos administrativos. 


\section{Conclusiones}

El objetivo de la investigación fue explicar la incidencia de las dimensiones de la contabilidad de gestión en los factores clave del éxito competitivo de las cooperativas multiactivas de Barranquilla (Colombia), el cual, toma relevancia debido a la importante participación de estas entidades en la economía del país. Se demuestra la influencia positiva y significativa de la amplitud, oportunidad e integración sobre los factores clave del éxito competitivo. Estos hallazgos destacan la importancia de generar información contable que, en el contexto de la teoría de los recursos y capacidades, contribuya al desarrollo de ventajas competitivas, a través de la identificación de los factores clave del éxito competitivo; reduciendo la incertidumbre en la toma de decisiones.

Los resultados contribuyen a la literatura proponiendo una razón por la que se presenta el vacío teórico entre los aportes de Isa y Foong (2005), Ismail e Isa (2011) y Poporato (2015); ya que evidencia la contribución de la contabilidad de gestión a los factores clave del éxito competitivo a través de la confirmación de las $\mathrm{H}_{1}, \mathrm{H}_{2} \mathrm{y} \mathrm{H}_{3}$ y explica por qué retrasa la capacidad de respuesta de las empresas, al identificar la influencia significativa y negativa de la agregación $\left(\mathrm{H}_{4}\right)$. Lo anterior permite deducir, al menos tentativamente que, en el contexto del estudio resulta más costoso aplicar sistemas de información agregados que desistir de ellos, debido a que la agregación es la dimensión de la contabilidad de gestión de mayor complejidad, al propender por revelar información detallada en líneas básicas de producto, departamentos o periodos de tiempo, lo que la hace más costosa y difícil de implementar; así como mayores requerimientos de tiempo para prepararla de manera oportuna, confiable y agregada.

En efecto, al confirmarse $\mathrm{H}_{1}, \mathrm{H}_{2} \mathrm{y}_{3}$ se ratifica la utilidad que debe poseer la información contable para sustentar el desarrollo de estrategias competitivas. Por lo tanto, se sugiere a los directivos de las cooperativas multiactivas que, centren sus esfuerzos en la manipulación de variables como la amplitud, oportunidad e integración de la información, ya que, marcaron una incidencia significativa y positiva en los factores clave del éxito competitivo.

El estudio presenta limitaciones que constituyen oportunidades para futuras líneas de investigación, debido a que, la elección de las cooperativas multiactivas de Barranquilla como unidades muestrales, aunque ha permitido profundizar en sus particularidades, implica en una generalización más amplia de los resultados, ya que el sector solidario se encuentra integrado, además, por instituciones mutualistas y asociativas, las cuales poseen características distintivas. Por tal motivo, se sugiere extender el estudio hacia estas entidades. Por otra parte, se considera oportuno desarrollar investigaciones cuyo diseño sean longitudinales con datos de panel, a fin de tener mayor robustez 
en los datos obtenidos y profundizar en el análisis de la relación negativa de la agregación con los factores clave de éxito competitivo.

Debido a que el objetivo de la contabilidad de gestión es la satisfacción de necesidades de usuarios internos y no la generación de informes de propósito general, esta es elaborada a discreción de cada empresa y presenta matices particulares, por tanto, se recomienda desarrollar investigaciones complementarias que la aborden a través de enfoques cualitativos, que permitan interpretar las subjetividades de los directivos y de los preparadores de la información.

\section{Referencias bibliográficas}

ACOSTA, M. (1999): Factores internos y competitividad, Tesis Doctoral, Universidad de la Laguna, Santa Cruz de Tenerife, España.

AGUIAR, T., MORENO, S. \& PICAZO, P. (2016): "How could traditional travel agencies improve their competitiveness and survive? A qualitative study in Spain", Tourism Management Perspectives, 20, 98-108. DOI: 10.1016/j.tmp.2016.07.011.

ANGELES, R. (2010): "Moderated multiple regression of absorptive capacity attributes and deployment outcomes: The importance of RFID IT infrastructure integration and supply chain process integration", International Journal of Information Systems and Supply Chain Management, 3(2), 25-51. DOI: 10.4018/jisscm.2010040102.

ANHEIR, H. \& SEIBEL, W. (1990): The Third sector comparative studies of nonprofit organizations, Berlin: De Gruyten.

ARCAS, N., GARCÍA-MARTÍNEZ, G. \& MELIÁ-MARTÍ, E. (2013): "El tamaño de las cooperativas agroalimentarias como factor de competitividad. Análisis de las fórmulas de crecimiento para alcanzarlo". En: Tamaño y Competitividad. Experiencias de crecimiento de las cooperativas agroalimentarias españolas, 25-66, Murcia: Cajamar Caja Rural.

BARNEY, J. (1991): "Firm resources and sustained competitive advantage", Journal of Management, 17(1), 99-120. DOI: 10.1177/014920639101700108.

BENS, D., MONAHAN, S. \& STEELE, L. (2018): "The effect of aggregation of accounting information via segment reporting on accounting conservatism", European Accounting Review, 27(2), 237262. DOI: $10.1080 / 09638180.2016 .1260488$. 
BERNAL, C., FRACICA, G. \& FROST, J. (2012): "Análisis de la relación entre la innovación y la gestión del conocimiento con la competitividad empresarial en una muestra de empresas en la ciudad de Bogotá", Estudios gerenciales, 28, 303-315. DOI: 10.18046/j.estger.2012.1490.

BLOODGOOD, J. (2019): "Knowledge acquisition and firm competitiveness: the role of complements and knowledge source", Journal of Knowledge Management, 23(1), 46-66. DOI: 10.1108/JKM-092017-0430.

BOUWENS, J. \& ABERNETHY, M. (2000): "The consequenses of customization of management accounting system desing", Accounting, Organization and Society, 25, 221-241. DOI: 10.1016/ S0361-3682(99)00043-4.

BRUQUE, S., HERNÁNDEZ, J., MOYANO, J. \& VARGAS, A. (2002): “¿Son más competitivas las sociedades cooperativas? Un análisis en el sector de la distribución", CIRIEC-España, Revista de Economía Pública, Social y Cooperativa, 42, 131-157.

CALDERA, J., BAUJIN, P., RIPOLL, V. \& VEGA, V. (2007): "Evolución en la Configuración de los Sistemas de Costeo Basado en las Actividades", Actualidad contable FACES, 10(14), 13-28.

CAMILLUS, J. \& DATTA, D. (1991): "Managing strategic issues in a turbulent environment", Long Range Planning, 24(2), 67-74, DOI: 10.1016/0024-6301(91)90081-X.

CHEN, J., CHENG, X., GONG, S. \& TAN, Y. (2020): "Project-level disclosure and investment efficiency: Evidence from China", Journal of Accounting, Auditing and Finance, Article in press. DOI: https://doi.org/10.1177/0148558X20912099.

CHENHALL, R. \& MORRIS, G. (1986): "The impact of structure, enviroment and interdependance on the perceived usefulness of management accounting system", The accounting review, 61(1), 16-35.

COMITÉ ECONÓMICO Y SOCIAL EUROPEO (2012): La Economía Social de la Unión Europea, Bruselas: Unidad de Visitas y Publicaciones.

Confederación de Cooperativas de Colombia (CONFECOOP) (2017): Cooperativas: Empresas para la gestión social y económica, Bogotá D.C: Diseño Editorial El Altillo.

CONGRESO DE LA REPÚBLICA DE COLOMBIA (1988): Ley 79 del 23 de diciembre de 1988, por medio del cual se actualiza la legislación cooperativa, Bogotá D.C. Diario oficial 38648 de Enero 10 de 1989.

CONGRESO DE LA REPÚBLICA DE COLOMBIA (2004): Ley 905 de 2004 "Por medio de la cual se modifica la Ley 590 de 2000 sobre promoción del desarrollo de la micro, pequeña y mediana empresa colombiana y se dictan otras disposiciones", Bogotá D.C., Colombia: Diario Oficial 45628. 
CRESPO, N., RODRIGUES, R., SAMAGAIO, A. \& MIRANDA, G. (2019): "The adoption of management control systems by start-ups: Internal factors and context as determinants", Journal of Business Research, 101, 875-884. DOI: 10.1016/j.jbusres.2018.11.020.

CRUZ-CÁZARES, C., BAYONA-SÁEZ, C., GARCÍA-MARCO, T., BERENDS, H., SMITS, A. \& REYMEN, I. (2018): "Public funds and internal innovation goals as drivers of formal and informal open innovation practices: A European regional comparison", Management Research, 16(2), 159-178. DOI: 10.1108/MRJIAM-03-2017-0739.

DEL ORDEN, O. \& GARMENDINA, A. (2008): Estructura de propiedad y grupos empresariales en España y la CAPV, Bilbao: Orkestra, Instituto Vasco de Competitividad.

DÍAZ, J. \& MÁRQUEZ, J. (2005): "Formación del recurso humano: visión tradicional y visión por competencias", Omnia, 11(2), 75-91.

ENRÍQUEZ, L. DE GUADALUPE, C. \& RODRÍGUEZ, R. (2013): "La gestión del conocimiento como elemento de competitividad en la industria manufacturera en Aguascalientes", Global Conference on Finance Proceeding, 8(1), 642-658.

FOMIN, V. \& POTOKINA, E. (2020): "Trends in Optimizing the Formation of Consolidated Reporting in Holding Companies in the Context of Global Digitization", Lecture Notes in Networks and Systems, 84, 233-242. DOI:10.1007/978-3-030-27015-5_29.

GAJARATI, D. \& PORTER, D. (2009): Econometría, México D.F.: Mc Graw Hill.

GARCÍA, M. \& LÓPEZ, V. (2014): "Evaluación de la competitividad de los restaurantes de cocina internacional", Revista Internacional Administracion \& Finanzas, 7(1), 79-88.

GHASEMI, R., AZMI, N., KARAMI, M., HAFIZ, N. \& ASGHARIZADE, E. (2015): "The Relationship among Strategy, Competition and Management Accounting Systems on Organizational Performance", European Online Journal of Natural and Social Sciences, 4(3), 565-581.

GOVDYA, V. \& KHROMOVA, I. (2018): "Methodical aspects of the decomposition approach to the formation of the managerial cost accounting system in the organizations of the Russian agroindustrial complex", Journal of Applied Economic Sciences, 13(3), 830-873.

GRANT, R. (2003): "Strategic planning in a turbulent environment: Evidence from the oil majors", Strategic Management Journal, 24(6), 491-517. DOI: 10.1002/smj.314.

HASSAN, A., ADHIKARIPARAJULI, M., FLETCHER, M. \& ELAMER, A. (2019): "Integrated reporting in UK higher education institutions", Sustainability Accounting, Management and Policy Journal, 10(5), 844-876. DOI: 10.1108/SAMPJ-03-2018-0093.

HERNÁNDEZ, R., FERNÁNDEZ, C. \& BAPTISTA, P. (2014): Metodología de la investigación (Sexta ed.), México D.F: Mc Graw Hill. 
HONG, P., LEE, S., MYNATT, P., \& RAMAKRISHNAN, R. (2019): "The value relevance of timely information: The case of comparable store sales growth", Advances in Accounting, 44, 10-21. DOI: 10.1016/j.adiac.2018.11.002.

ISA, C. \& FOONG, S. (2005): "Adoption of advanced manufacturing technology (AMT) and management accounting practices: the case of manufacturing firms in Malaysia", World Review of Science, Technology and Sustainable Development, 2(1), 35-48. DOI: 10.1504/WRSTSD.2005.006726.

ISMAIL, K. \& ISA, C. (2011): "The Role of Management Accounting Systems In Advanced Manufacturing Environment", Australian Journal of Basic and Applied Sciences, 5(9), 2196-2209.

ISMAIL, K., RUHANA, C. \& MIA, L. (2018): "Market Competition, Lean Manufacturing Practices and The Role of Management Accounting Systems (MAS) Information", Jurnal Pengurusan, 52, 47-61. DOI: 10.17576/pengurusan-2018-52-04.

JOHNSON, H. \& KAPLAN, R. (1987): Relevance lost: the rise and fall of management accounting, Boston: Harvard Business School Press.

KAJÜTER, P. \& NIENHAUS, M. (2017): "The Impact of IFRS 8 Adoption on the Usefulness of Segment Reports", Abacus, 53(1), 28-58. DOI: 10.1111/abac.12105.

KATTAN, F., PIKE, R. \& TAYLES, M. (2007): "Reliance of management accounting under environmental uncertainty: The caseof Palestine", Journal of Accounting \& Organizational Change, 3(3), 227-249. DOI: $10.1108 / 18325910710820283$.

LADRÓN, R., VARGAS, A., BLANCO, L., ROA, E., CÁCERES, L. \& VARGAS, L. (2018): "Características de la economía solidaria colombiana. Aproximaciones a las corrientes influyentes en Colombia", CIRIEC-España, Revista de Economía Pública, Social y Cooperativa, 93, 85-113. DOI: 10.7203/CIRIEC-E.93.10327.

LAJARA-CAMILLERI, N. \& SERVER-IZQUIERDO, R. (2017): “¿Cómo se puede mejorar la competitividad de las cooperativas agroalimentarias?", CIRIEC-España, Revista de Economía Pública, Social y Cooperativa, 90, 103-121. DOI:10.7203/CIRIEC-E.90.8854.

LIAO, C., CHEN, Y. \& TANG, C. (2019): "Information provision policies for improving farmer welfare in developing countries: Heterogeneous farmers and market selection", Manufacturing and Service Operations Management, 21(2), 254-270. DOI: 10.1287/msom.2016.0599.

LLORENTE, I. (2013): Análisis de competitividad de las empresas de acuicultura. Aplicaciones empiricas al cultivo de la Dorada (Sparus Auratha) y la lubina (Dicentrarchus labrax), Tesis Doctoral, Universidad de Cantabria, Santander, España.

LÓPEZ, M. (2007): Planeación estratégica de tecnologías informáticas y sistemas de información, Caldas: Universidad de Caldas. 
LÓPEZ-GAMERO, M. \& MOLINA-AZORÍN, J. (2016): "Environmental Management and Firm Competitiveness: The Joint Analysis of External and Internal Elements", Long Range Planning, 49(6), 746-763. DOI: 10.1016/.J.rp.2015.12.002.

LÓPEZ-GAMERO, M., MOLINA-AZORÍN, J. \& CLAVER-CORTÉS, E. (2010): "The potential of environmental regulation to change managerial perception, environmental management, competitiveness and financial performance", Journal of Cleaner Production, 18(10-11), 963-974. DOI: 10.1016/j.jclepro.2010.02.015.

LÓPEZ-VALEIRAS, E., GONZÁLEZ-SÁNCHEZ, M. \& GÓMEZ-CONDE, J. (2016): "The effects of the interactive use of management control systems on process and organizational innovation", Review of Managerial Science, 10(3), 487-510. DOI: 10.1007/s11846-015-0165-9.

MANGALISO, M. (1995): "The strategic usefulness of management information as perceived by middle managers", Journal of Management, 21(2), 231-250. DOI: 10.1177/014920639502100204.

MANYAEVA, V., PISKUNOV, V. \& FOMIN, V. (2016): "Strategic management accounting of company costs", International Review of Management and Marketing, 6(5), 255-264.

MARTÍNEZ, R., CHARTERINA, J. \& ARAUJO, A. (2010): "Un modelo causal de competitividad empresarial planteado desde la VBR: capacidades directivas, de innovación, marketing y calidad", Investigaciones Europeas de Dirección y Economía de la Empresa, 16(2), 165-188. DOI: 10.1016/ S1135-2523(12)60117-8.

MENDOZA, J. (2013): "La capacidad dinámica de ripostar en la empresa: confrontar entornos volátiles", Cuadernos de Administración, 26(47), 63-85.

MOGROVEJO, R., MORA, A. \& VANHUYNEGEM, P. (2012): El cooperativismo en América Latina: Una diversidad de contribuciones al desarrollo sostenible, La Paz: Oficina Internacional del Trabajo (OIT): Oficina de la OIT para los Países Andinos.

MOLL, J. \& YIGITBASIOGLU, O. (2019): "The role of internet-related technologies in shaping the work of accountants: New directions for accounting research", British Accounting Review, 51(6), 4-20. DOI: 10.1016/j.bar.2019.04.002.

MONTEGUT, Y. (2006): Análisis de los factores explicativos del éxito competitivo en las almazaras cooperativas catalanas, Tesis doctoral, Universitat de Lleida, España.

MORA, E. (2013): Nivel de competitividad de las Mipymes de Bogotá. Análisis por dimensiones estratégicas, Tesis de Maestría, Universidad Nacional de Colombia, Bogotá, Colombia.

MORIANO, A. (2008): "Cooperativas multiactivas". En: J. Alvárez, Naturaleza y especificidad de la cooperativa multiactiva, Bogotá: COOTRADIAN - Escuela de Economía Solidaria, 5-18.

MOYANO, J., PUIG, F. \& BRUQUE, S. (2008): "Los determinantes de la competitividad en las cooperativas", CIRIEC-España, Revista de Economía Pública, Social y Cooperativa, 61, 233-249. 
NARANJO, D. (2005): "El papel de los estilos de gestión y sistemas contables de gestión en el cambio estratégico", Revista Española de Financiación y Contabilidad, 34(127), 951-975. DOI: 10.1080/02102412.2005.10779568.

NARANJO-GIL, D. \& HARTMANN, F. (2007): "Management accounting systems, top management team heterogeneity and strategic change", Accounting, Organizations and Society, 32(7), 735756, DOI: 10.1016/j.aos.2006.08.003.

NILSSON, J., KIHLEN, A. \& NORELL, L. (2009): "Are Traditional Cooperatives an Endangered species? About Shrinking Satisfaction, Involvement and Trust", International food and agribusiness, 12(4), 101-120.

PAL, R. \& ALTAY, N. (2019): "Identifying Key Success Factors for Social Enterprises Serving Base-of-Pyramid Markets through Analysis of Value Chain Complexities", Journal of Business Logistics, 40(2), 161-179, DOI:10.1111/jbl.12212.

PARILINA, E. \& SEDAKOV, A. (2020): "Stable coalition structures in dynamic competitive environment", International Series in Operations Research and Management Science, 280, 381-396, DOI: 10.1007/978-3-030-19107-8_21.

PAZ, S., AULICINO, J. \& PEREYRA, A. (2013): "Competitividad de los haras de sangre pura de carrera en función de los factores clave de éxito", Archivos de zootecnia, 62(239), 333-344, DOI: 10.4321/S0004-05922013000300002.

PENROSE, E. (1959): The theory of the growth of the firm basic, Londres: Basic Blackwell.

PORPORATO, M. (2015): "Contabilidad de gestión para controlar o coordinar en entornos turbulentos: su impacto en el desempeño organizacional", Contaduría y Administración, 60, 511-534, 10.1016/j.cya.2015.02.002.

PORTER, M. (1991): La ventaja competitiva de las naciones, Barcelona, España: Vergara.

PORTER, M. (2008): "Las cinco fuerzas competitivas que le dan forma a la estrategia", Harvard Business Review, 86(1), 58-77.

PRIETO, B., SANTIDRIÁN, A. \& VALLADARES, H. (2007): "El sistema ABC en el sector logístico mexicano. Un análisis empírico", Revista iberoamericana de contabilidad de gestión, 10, 13-56.

PRODANOVA, N., DAVYDOVA, A., SOTNIKOVA, L., SHEVCHENKO, S., BOCHKAREVA, N. \& POLYANSKAYA, T. (2019): "Fundamental approaches for the formation of integrated corporate reporting", International Journal of Economics and Business Administration, 7(3), 293-304, DOI: 10.35808/ijeba/326.

RUBIO, M. \& UNSAL, D. (2020): "Macroprudential policy under incomplete information", European Journal of Finance, 26(7-8), 616-639, DOI: 10.1080/1351847X.2019.1679209. 
SAAVEDRA, M. (2012): Una propuesta para la determinación de la competitividad en la pyme latinoamericana. Pensamiento y gestión, 33, 93-124.

SHAROVATOVA, E., CHUKHROVA, O., SCHERBAKOVA, E. \& MARTIROSYAN, T. (2018): "Innovative production accounting segmentation in a globalized economy", European Research Studies Journal, 21, 540-550.

SILVA, J. (2008): "La multiactividad en números". En: J. Alvárez, Naturaleza y especificidad de la cooperativa multiactiva, 115-124. Bogotá D.C. COOTRADIAN - Escuela de Economía Solidaria.

SIMONS, R. (1990): "The role of management control systems in creating competitive advantage: New perspectives", Accounting, Organizations and Society, 15(1-2), 127-143. DOI: 10.1016/03613682(90)90018-P.

SIMONS, R. (1995): Levers of Control: How Managers Use Innovative Control Systems to Drive Strategic Renewal, Boston: Harvard Business School Press.

SIQANI, S. \& VOKSHI, N. (2019): "The impact of accounting information system on the effectiveness of public enterprises: The case of Kosovo", International Journal of Economics and Business Administration, 7(3), 106-115.

SOLLEIRO, J. \& CASTAÑÓN, R. (2012): "Competitividad, innovación y transferencia de tecnología en México", Innovación y competitividad, 869, 149-161.

TERDPAOPONG, K., VISEDSUN, N. \& NITIROJNTANAD, K. (2019): "Value creation through management accounting practices in large thai manufacturing companies", Polish journal of management studies, 395-406. DOI: 10.17512/pjms.2019.19.2.34.

THEEUWES, J. \& ADRIAANSEN, J. (1994): "Towards an integrated accounting framework for manufacturing improvement", International Journal of Production Economics, 36(1), 85-96. DOI: 10.1016/0925-5273(94)90151-1.

THOMSEN, S. (1995): "Using online databases in corporate issues management", Public Relations Review, 21(2), 103-122, DOI: 10.1016/0363-8111(95)90002-0.

TWEDT, B. (2016): "Spreading the Word: Price Discovery and Newswire Dissemination of Management Earnings Guidance", Accounting Review, 45(4), 425-436. DOI: 10.2308/accr-51129.

VÁZQUEZ, G., GUERRERO, J. \& NÚÑEZ, T. (2014): "Gestión de conocimiento, capital intelectual y competitividad en pymes manufactureras en México", Retos, 7(1), 29-43.

VELANDIA, G. (2010): "Un concepto sobre la cientificidad de la contabilidad", Adversia, 5, 110-127.

VELANDIA, G., ANGUILA, A. \& ARCHIBOLD, W. (2018): "La contabilidad como tecnología blanda: una visión desde los imaginarios sociales", Cuadernos de Contabilidad, 18(45), 10.11144/Javeriana.cc18-45.ctvi. 
VELANDIA, G., HERNÁNDEZ, L., PORTILLO, R., ALVEAR, L. \& CRISSIEN, T. (2016): "Rasgos de la administración de la microempresa en Barranquilla Colombia", Espacios, 37(9), 7-22.

VILLAFAÑEZ, I. (2015): "El deber de lealtad y sus concreciones en la legislación cooperativa", REVESCO, Revista De Estudios Cooperativos, 120, 121-148. DOI: 10.5209/rev_REVE.2016. v120.49698.

WEISS, A., LURIE, N. \& MACINNIS, D. (2008): "Listening to strangers: Whose responses are valuable, how valuable are they and why?", Journal of Marketing Research, 45(4), 425-436. DOI: 10.1509/jmkr.45.4.425.

WERNERFELT, B. (1984): "A Resource-Based View of the Firm", Strategic Management Journal, 5(2), 171-180. DOI: 10.1002/smj.4250050207. 